\title{
Investigating Effect of Cerium (IV) Sulfate Tetrahydrate as Reusable and Heterogeneous Catalyst for the One-pot Multicomponent Synthesis of Polyhydroquinolines
}

\author{
Elham kazemia , Abolghasem Davoodniaa,", Samira Basafaa , Ahmad Nakhaei ${ }^{\text {b,*, }}$ \\ Niloofar Tavakoli-Hoseini ${ }^{\mathrm{b}}$
}

\author{
${ }^{a}$ Department of Chemistry, Mashhad Branch, Islamic Azad University, Mashhad, Iran \\ ${ }^{\mathrm{b}}$ Young Researchers and Elite Club, Mashhad Branch, Islamic Azad University, Mashhad, Iran \\ *Corresponding authors: E-mail address: (adavoodnia@mshdiau.ac.ir, adavoodnia@yahoo.com), \\ (nakhaei_a@yahoo.com, nakhaei_a@mshdiau.ac.ir), Tel.: +989370355756
}

Received: 23 October 2018, Revised: 12 November 2018, Accepted: 27 November 2018

\begin{abstract}
A B S T R A C T
Cerium (IV) sulfate tetrahydrate, $\mathrm{Ce}\left(\mathrm{SO}_{4}\right)_{2} .4 \mathrm{H}_{2} \mathrm{O}$ used as a new inorganic solid acidic catalyst for the synthesis of polyhydroquinoline derivatives via four-component Hantzsch reaction of aromatic/aliphatic aldehydes, 5,5-dimethylcyclohexane-1,3-dione (dimedone), ethyl acetoacetate and ammonium acetate under solvent-free conditions at $120{ }^{\circ} \mathrm{C}$ was investigated. This method has the advantages of high yields (88-97\%), clean reaction, simple methodology, and short reaction time (15-25 min). However, the aromatic aldehydes whether electron donating or electron withdrawing showed simple transformation with the excellent yields than the other aliphatic. Furthermore, the catalyst is inexpensive and readily available and can be recovered conveniently and reused efficiently without considerable decrease in its catalytic activity.
\end{abstract}

Keywords: Cerium (IV) sulfate tetrahydrate, Polyhydroquinolines, Heterogeneous catalyst, Hyantzsch reaction, One-pot multicomponent.

\section{G R A P H I C A L A B S T RACT}

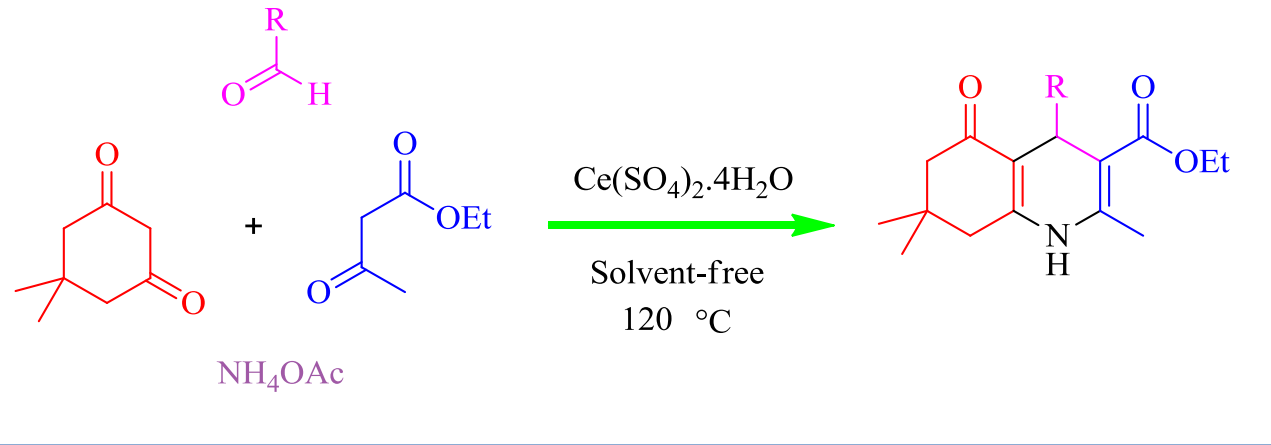




\section{Introduction}

Polyhydroquinolines (PHQs), nitrogenbased heterocyclic compounds with 1,4dihydropyridine skeleton, demonstrate a variety of biological activities such as antiasthmatic, anti-inflammatory, antimalarial, anticancer, antidiabetic and anthelmintic agents [1]. Numerous methods for the preparation of polyhydroquinoline derivatives have been reported [2-7]. The classical methods include the threecomponent condensation of an aldehyde with ethyl acetoacetate, and ammonia in acetic acid or in refluxing alcohol [4]. A number of new efficient methods have been developed including the use of Metal Oxide Nanoparticles (MON) [3], microwave [5], sonication [6] and a variety of different catalysts [8-21].

However, many of these methods have disadvantages such as high temperatures, protracted reaction time, tedious work-up, acidic or basic catalysts and the use of relatively expensive reagents. Moreover, the main drawback of nearly all existing methods is that the catalysts are destroyed in the work-up procedure and cannot be recovered or reused. Therefore, the studies attempt to detect a new and better catalyst for the synthesis of organic compounds in terms of economic, high activity, greater selectivity and reusability [22-26].
Cerium (IV) salts have been pointed out lately as ease of product separation, recycling of the catalyst, and environmental acceptability compared with various inorganic salts [27]. These compounds have been used for many organic chemistry such as chemoselective deprotection of tetrahydropyranyl, methoxymethyl, and benzyloxymethyl ethers [27], synthesis of acetamido phenols promoted by $\mathrm{Ce}\left(\mathrm{SO}_{4}\right)_{2}$ [28] and conversion of oximes into aldehydes and ketones [29]. According to our knowledge there are no examples of the use of $\mathrm{Ce}\left(\mathrm{SO}_{4}\right)_{2} \cdot 4 \mathrm{H}_{2} \mathrm{O}$ as catalyst for the synthesis of polyhydroquinolines.

According to our previous published works, around the improving organic reactions in the presence of various catalysts [30-46], in this study, we have developed and optimized a catalyzed process of Polyhydroquinoline via Hantzsch reaction. These compounds 5a-l were synthesized from the four-component reaction of 5,5dimethyl-1,3- cyclohexaedione1, different aldehydes 2a-l, ethyl acetoacetate 3, and ammonium acetate $\mathbf{4}$ under solvent-free conditions using $\mathrm{Ce}\left(\mathrm{SO}_{4}\right)_{2} \cdot 4 \mathrm{H}_{2} \mathrm{O}$ as a new and reusable inorganic solid acidic catalyst.

\section{Experimental}

\section{Methods and apparatus}

The chemicals were purchased from Merck and Aldrich and used without further 
purification. The monitoring of the reaction and purity determination of the products were accomplished by TLC. The melting points were recorded using a Stuart SMP3 melting point apparatus. The IR spectra were obtained using a Tensor 27 Bruker spectrophotometer in $\mathrm{KBr}$ disks. The ${ }^{1} \mathrm{H}$ NMR spectra were recorded using Bruker $400 \mathrm{MHz}$ spectrometers.

\section{General procedure for the synthesis of} polyhydroquinolines $5 a-1$

A mixture of dimedone 1 (1 $\mathrm{mmol})$, different aldehydes 2a-l (1 mmol), ethyl acetoacetate 3 ( $1 \mathrm{mmol}$ ), ammonium acetate $4(1 \mathrm{mmol})$ and $\mathrm{Ce}\left(\mathrm{SO}_{4}\right)_{2} .4 \mathrm{H}_{2} \mathrm{O}(0.1 \mathrm{mmol}, 10$ mol\%) was heated in an oil bath at $120^{\circ} \mathrm{C}$ for 15-25 min. The monitoring of the reaction and purity determination of the products were accomplished by TLC. Upon completion of the transformation, the reaction mixture was cooled to room temperature, and hot ethanol (5 mL) was added. The precipitated catalyst was collected by filtration. Also, the resulting solid product was collected and recrystallized from ethanol to give the net product 5a-1 (Scheme 1).

\section{Selected spectroscopic data}

Ethyl 4-(2-chloro phenyl)-2,7,7trimethyl-5-oxo-

1,4,5,6,7,8hexahydroquinoline-3-

carboxylate (5c): IR (KBr): $v_{\max }$ : 3282, 3199, 3077, 2957, 1698, 1610, 1494, $1380,1216,752 \mathrm{~cm}^{-1}$; ${ }^{1} \mathrm{H}-\mathrm{NMR}(400 \mathrm{MHz}$, $\left.\mathrm{CDCl}_{3}\right) \delta: 0.96\left(\mathrm{~s}, 3 \mathrm{H}, \mathrm{CH}_{3}\right), 1.1(\mathrm{~s}, 3 \mathrm{H}$, $\left.\mathrm{CH}_{3}\right), 1.2\left(\mathrm{t}, 3 \mathrm{H}, J=7.2 \mathrm{~Hz}, \mathrm{CH}_{3}\right), 1.99-2.2$ $\left(\mathrm{m}, 4 \mathrm{H}, 2 \mathrm{CH}_{2}\right), 2.3\left(\mathrm{~s}, 3 \mathrm{H}, \mathrm{CH}_{3}\right), 4.05(\mathrm{t}$, $\left.2 \mathrm{H}, J=7.2 \mathrm{~Hz}, \mathrm{CH}_{2}\right), 5.4(\mathrm{~s}, 1 \mathrm{H}, \mathrm{CH}), 6.1(\mathrm{~s}$, $1 \mathrm{H}, \mathrm{NH}), 7.04-7.4(\mathrm{~m}, 4 \mathrm{H}$, arom-H).

Ethyl 4-(4-bromophenyl)-2,7,7trimethyl-5-oxo-1,4,5,6,7,8-

hexahydroquinoline-3- carboxylate (5d): IR (KBr): $v_{\text {max }}$ 3275, 3206, 3076, 2958, 1703, 1604, 1487, 1381, 1215, $842 \mathrm{~cm}^{-1}$; ${ }^{1} \mathrm{H}-\mathrm{NMR}\left(400 \mathrm{MHz}, \mathrm{CDCl}_{3}\right) \delta: 0.95(\mathrm{~s}, 3 \mathrm{H}$, $\left.\mathrm{CH}_{3}\right), 1.09\left(\mathrm{~s}, 3 \mathrm{H}, \mathrm{CH}_{3}\right), 1.21(\mathrm{t}, 3 \mathrm{H}, J=7.2$ $\mathrm{Hz}, \mathrm{CH}_{3}$ ), 2.14-2.37 (m, 4H, 2CH $\left.\mathrm{CH}_{2}\right) 2.39$ (s, $\left.3 \mathrm{H}, \mathrm{CH}_{3}\right), 4.06\left(\mathrm{q}, 2 \mathrm{H}, J=7.2 \mathrm{~Hz}, \mathrm{OCH}_{2}\right.$ ), 5.03 (s, 1H, CH) 5.95 (s, 1H, NH), 5.95 (s, $1 \mathrm{H}, \mathrm{NH}), 7.21(\mathrm{~d}, J=8.4 \mathrm{~Hz}, 2 \mathrm{H}$, arom-H), $7.34(\mathrm{~d}, J=8.4 \mathrm{~Hz}, 2 \mathrm{H}$, arom-H).

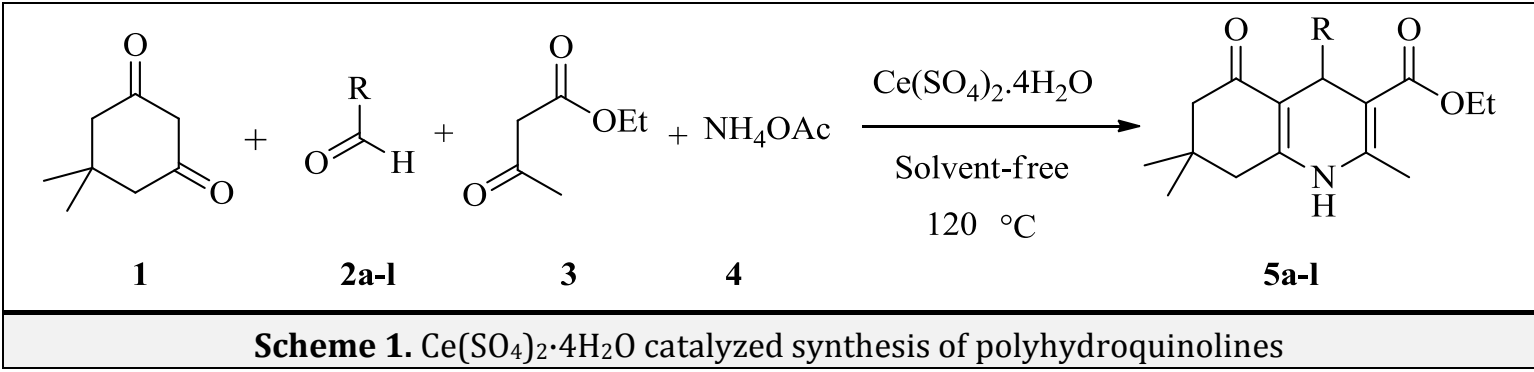


Ethyl 4-(3- hydroxyphenyl)-2,7,7-trimethyl5-oxo-1,4,5,6,7,8hexahydroquinoline-3carboxylate (5f): IR (KBr): $v_{\max }$ 3278, 3083, 2960, 1685, 1612, 1488, 1379, 1274, 1216, $695 \mathrm{~cm}^{-1}$; ${ }^{1} \mathrm{H}-\mathrm{NMR}$ (400 MHz, DMSO) $\delta: 0.87$ $\left(\mathrm{s}, 3 \mathrm{H}, \mathrm{CH}_{3}\right), 1\left(\mathrm{~s}, 3 \mathrm{H}, \mathrm{CH}_{3}\right), 1.15(\mathrm{t}, 3 \mathrm{H}, J=7.2$ $\left.\mathrm{Hz}, \mathrm{CH}_{3}\right), 1.97-2.5\left(\mathrm{~m}, 4 \mathrm{H}, 2 \mathrm{CH}_{2}\right), 4(\mathrm{q}, 2 \mathrm{H}, J=$ $7.2 \mathrm{~Hz}, 2 \mathrm{CH}_{2}$ ), 4.8 (s, 1H, CH), 6.4 (m., $1 \mathrm{H}$, arom-H), 6.6 (d, J = 7.2 Hz, 2H, arom-H), 6.9 $(\mathrm{t}, J=7.2 \mathrm{~Hz}, 1 \mathrm{H}$, arom-H), $9(\mathrm{~s}, 1 \mathrm{H}, \mathrm{OH}$ or $\mathrm{NH}), 9.1$ (s, 1H, OH or $\mathrm{NH})$.

Ethyl 4-(3-nitrophenyl)-2,7,7-trimethyl-5oxo-1,4,5,6,7,8 hexahydroquinoline-3carboxylat (5h): IR (KBr): $v_{\max }$ 3283, 2958, 2928, 1704, 1606, 1534, 1486, 1351, 1211, $681 \mathrm{~cm}^{-1} ;{ }^{1} \mathrm{H}-\mathrm{NMR}\left(400 \mathrm{MHz}, \mathrm{CDCl}_{3}\right) \delta: 0.95$ $\left(\mathrm{s}, 3 \mathrm{H}, \mathrm{CH}_{3}\right), 1.1\left(\mathrm{~s}, 3 \mathrm{H}, \mathrm{CH}_{3}\right), 1.21(\mathrm{t}, 3 \mathrm{H}, J=$ $7.2 \mathrm{~Hz}, \mathrm{CH}_{3}$ ), 2.1-2.38 (m, 4H, 2CH 2 ), 2.41 (s, $3 \mathrm{H}, \mathrm{CH}_{3}$ ), $4.06\left(\mathrm{q}, 2 \mathrm{H}, J=7.2 \mathrm{~Hz}, \mathrm{CH}_{2}\right.$ ), 5.2 (s, $1 \mathrm{H}, \mathrm{CH}), 6.2(\mathrm{~s}, 1 \mathrm{H}, \mathrm{NH}), 7.4-8.13(\mathrm{~m}, 4 \mathrm{H}$, arom-H).

\section{Results and Discussion}

In the present research, in order to find out the optimal conditions such as temperature and amount of $\mathrm{Ce}\left(\mathrm{SO}_{4}\right)_{2} .4 \mathrm{H}_{2} \mathrm{O}$, a model reaction was selected for the synthesis of compound $\mathbf{5 b}$ with dimedone $\mathbf{1}$ (1mmol), 4chlorobenzaldehyde 2b (1mmol), ethyl acetoacetate 3 (1mmol), and ammonium acetate 4 (1 $1 \mathrm{mmol})$ under solvent-free situation. The results are summarized in Table 1. From these observations, no product was observed, when the reaction was carried out under solvent-free conditions in the absence of catalyst, even after a long reaction time (entry 1 ). On the other hand, the presence of the $\mathrm{Ce}\left(\mathrm{SO}_{4}\right)_{2} .4 \mathrm{H}_{2} \mathrm{O}$ as catalyst has improved the yields of the reaction. Changing the percentage of the catalyst showed that 10 mol\% of $\mathrm{Ce}\left(\mathrm{SO}_{4}\right)_{2} .4 \mathrm{H}_{2} \mathrm{O}$ was adequate to push the reaction to completion within 15 min (entry 12). The $\mathrm{Ce}\left(\mathrm{SO}_{4}\right)_{2} .4 \mathrm{H}_{2} \mathrm{O}$ catalyzed reaction was investigated in different types of solvent. The product yield in refluxing EtOH, $\mathrm{CH}_{2} \mathrm{Cl}_{2}$ or $\mathrm{CH}_{3} \mathrm{CN}$ was low, even after prolonged reaction time (entries 17-19), whereas relatively good yields were obtained in refluxing $\mathrm{H}_{2} \mathrm{O}$ (entry 20). Therefore, the use of $10 \mathrm{~mol} \%$ of the catalyst at $120{ }^{\circ} \mathrm{C}$ under solvent free media were selected as optimum conditions (entry 12).

Under these conditions, yield of the $\mathrm{Ce}\left(\mathrm{SO}_{4}\right)_{2} .4 \mathrm{H}_{2} \mathrm{O} \quad$ via Hantzsch multicomponent condensation of a number of aldehyde with dimedone, ethyl acetoacetate and ammonium acetate were examined. The results are summarized in Table 2. As shown, aromatic aldehydes containing both electron donating and electron withdrawing groups reacted smoothly to produce the corresponding polyhydroquinolines in excellent yields over short time.

A comparison of our results with some 
other results reported for similar reactions has been tabulated in Table 3 to show the merit of the present method (except using Nano-Ni as a catalyst with microwave) [8]. As seen from the results, the rate of the reaction and yield of the current method were similar to or higher than those reported. Therefore, a number of the reported methodologies suffer from disadvantages such as high cost of the catalysts, the use of toxic reagents, use of halogenated solvents, the requirement for rigid reaction conditions and prolonged reaction time.
We also used the model reaction under optimized reaction conditions to evaluate the reusability of the catalyst. After completion of the reaction, the catalyst was recovered as described in the experimental section. The separated catalyst was washed with hot ethanol and subsequently dried at $50{ }^{\circ} \mathrm{C}$ under vacuum for $1 \mathrm{~h}$ before being reused in a similar reaction. We found that the catalyst could be used at least 5 times with only a slight reduction in activity (Figure 1).

Table 1. Optimization of the reaction conditions for the synthesis of compound $\mathbf{5 b}$ in the presence of $\mathrm{Ce}\left(\mathrm{SO}_{4}\right)_{2} \cdot 4 \mathrm{H}_{2} \mathrm{O}^{*}$

\begin{tabular}{|c|c|c|c|c|c|}
\hline Entry & Catalyst (mol\%) & Solvent & $\begin{array}{c}\text { Temperature } \\
\left({ }^{\circ} \mathrm{C}\right) \\
\end{array}$ & Time (min) & $\begin{array}{r}\text { Isolated } \\
\text { yield (\%) }\end{array}$ \\
\hline 1 & - & - & 120 & 60 & - \\
\hline 2 & 5 & - & 110 & 20 & 67.8 \\
\hline 3 & 5 & - & 120 & 15 & 86.5 \\
\hline 4 & 5 & - & 130 & 20 & 67.6 \\
\hline 5 & 7 & - & 90 & 20 & 38 \\
\hline 6 & 7 & - & 110 & 15 & 70.3 \\
\hline 7 & 7 & - & 120 & 15 & 89.9 \\
\hline 8 & 7 & - & 130 & 20 & 70.3 \\
\hline 9 & 10 & - & 80 & 20 & 62.2 \\
\hline 10 & 10 & - & 90 & 20 & 59.5 \\
\hline 11 & 10 & - & 110 & 15 & 75.5 \\
\hline 12 & 10 & - & 120 & 15 & 92 \\
\hline 13 & 10 & - & 130 & 20 & 89.2 \\
\hline 14 & 13 & - & 110 & 15 & 63 \\
\hline 15 & 13 & - & 120 & 15 & 59.5 \\
\hline 16 & 13 & - & 130 & 15 & 40.5 \\
\hline 17 & 10 & EtOH & Reflux & 90 & 48 \\
\hline 18 & 10 & $\mathrm{CH}_{2} \mathrm{Cl}_{2}$ & Reflux & 90 & 65 \\
\hline 19 & 10 & $\mathrm{CH}_{3} \mathrm{CN}$ & Reflux & 90 & 67 \\
\hline 20 & 10 & $\mathrm{H}_{2} \mathrm{O}$ & Reflux & 90 & 82 \\
\hline
\end{tabular}

*Reaction conditions: dimedone ( $1 \mathrm{mmol}), 4$-chlorobenzaldehyde $(1 \mathrm{mmol})$, ethyl acetoacetate $(1 \mathrm{mmol})$ and ammonium acetate $(1 \mathrm{mmol})$ 
Table 2. Synthesis of polyhydroquinolines $\mathbf{5 a - 5 1}$, catalyzed by $\mathrm{Ce}\left(\mathrm{SO}_{4}\right)_{2} \cdot 4 \mathrm{H}_{2} \mathrm{O}^{*}$

\begin{tabular}{|c|c|c|c|c|c|c|c|}
\hline \multirow{2}{*}{ Entry } & \multirow{2}{*}{$\mathbf{R}$} & \multirow{2}{*}{ Product } & \multirow{2}{*}{ Time (min) } & \multirow{2}{*}{$\begin{array}{c}\text { Isolated } \\
\text { yield (\%) }\end{array}$} & \multicolumn{2}{|c|}{ Melting point $\left({ }^{\circ} \mathrm{C}\right)$} & \multirow{2}{*}{ Ref. } \\
\hline & & & & & Found & Reported & \\
\hline 1 & $\mathrm{C}_{6} \mathrm{H}_{5}$ & $5 a$ & 15 & 95 & $216-218$ & $202-204$ & [17] \\
\hline 2 & $4-\mathrm{ClC}_{6} \mathrm{H}_{4}$ & $5 b$ & 15 & 92 & $246-248$ & $244-246$ & [11] \\
\hline 3 & $2-\mathrm{ClC}_{6} \mathrm{H}_{4}$ & $5 c$ & 15 & 91 & $208-210$ & 206-208 & [47] \\
\hline 4 & $4-\mathrm{BrC}_{6} \mathrm{H}_{4}$ & $5 d$ & 15 & 95 & $255-257$ & $259-260$ & [17] \\
\hline 5 & $3-\mathrm{BrC}_{6} \mathrm{H}_{4}$ & $5 e$ & 15 & 94 & $256-258$ & $258-260$ & [10] \\
\hline 6 & $3-\mathrm{OHC}_{6} \mathrm{H}_{4}$ & $5 f$ & 20 & 90 & $222-223$ & $225-227$ & {$[2]$} \\
\hline 7 & $4-\mathrm{O}_{2} \mathrm{NC}_{6} \mathrm{H}_{4}$ & $5 g$ & 25 & 97 & $250-251$ & $245-247$ & [47] \\
\hline 8 & $3-\mathrm{O}_{2} \mathrm{NC}_{6} \mathrm{H}_{4}$ & $5 \mathrm{~h}$ & 15 & 96 & $182-184$ & $178-180$ & [11] \\
\hline 9 & $4-\mathrm{MeC}_{6} \mathrm{H}_{4}$ & $5 \mathbf{i}$ & 15 & 92 & $264-266$ & $260-262$ & [17] \\
\hline 10 & $4-\mathrm{MeOC}_{6} \mathrm{H}_{4}$ & $5 \mathbf{j}$ & 15 & 95 & $259-260$ & $255-257$ & [17] \\
\hline 11 & Et & $5 \mathbf{k}$ & 25 & 88 & $165-167$ & $145-146$ & [2] \\
\hline 12 & $n-\operatorname{Pr}$ & 51 & 25 & 89 & $167-169$ & $146-147$ & [2] \\
\hline
\end{tabular}

*Reaction conditions: dimedone $\mathbf{1}(1 \mathrm{mmol})$, an aldehyde $\mathbf{2}(1 \mathrm{mmol})$, ethyl acetoacetate $\mathbf{3}(1 \mathrm{mmol})$, ammonium acetate $4(1 \mathrm{mmol}), \mathrm{Ce}\left(\mathrm{SO}_{4}\right)_{2} \cdot 4 \mathrm{H}_{2} \mathrm{O}\left(0.1 \mathrm{mmol}, 10 \mathrm{~mol} \%\right.$ based on aldehyde), $120{ }^{\circ} \mathrm{C}$, solventfree.

Table 3. Comparison of the synthesis of polyhydroquinolinesb with different catalysts

\begin{tabular}{|c|c|c|c|c|c|c|}
\hline Entry & Catalyst & Solvent & $\begin{array}{c}\text { Temperature } \\
\left({ }^{\circ} \mathrm{C}\right)\end{array}$ & $\begin{array}{l}\text { Time } \\
(\min )\end{array}$ & $\begin{array}{c}\text { Isolated } \\
\text { yield } \\
(\%)\end{array}$ & Ref. \\
\hline 1 & Nano-Ni & - & MW & $1-1.5$ & $85-96$ & [8] \\
\hline 2 & $\left(\mathrm{PPA}-\mathrm{SiO}_{2}\right)$ & - & 80 & $40-60$ & $85-92$ & [9] \\
\hline 3 & $\mathrm{Gd}(\mathrm{OTf})_{3}$ & - & r.t. & $300-330$ & $82-89$ & [10] \\
\hline 4 & $\begin{array}{l}\text { Bakers' yeast, D-glucose, } \\
\text { phosphate buffer }\end{array}$ & - & r.t. & 1440 & $62-84$ & [11] \\
\hline 5 & Trifluoroethanol (TFE) & TFE & 70 & 180 & $95-98$ & [12] \\
\hline 6 & $\mathrm{FeF}_{3}$ & EtOH & $75-80$ & $60-90$ & $85-95$ & [13] \\
\hline 7 & $\mathrm{Sc}(\mathrm{OTf})_{3}$ & EtOH & r.t. & $60-360$ & $85-95$ & [14] \\
\hline 8 & CAN & EtOH & r.t. & $25-240$ & $88-98$ & [15] \\
\hline 9 & $\mathrm{Yb}(\mathrm{OTf})_{3}$ & EtOH & r.t. & $60-480$ & $85-95$ & [17] \\
\hline 10 & $\mathrm{~K}_{7}\left[\mathrm{PW}_{11} \mathrm{CoO}_{40}\right]$ & $\mathrm{MeCN}$ & reflux & $25-30$ & $75-85$ & [16] \\
\hline 11 & $\mathrm{LaCl}_{3 .} 7 \mathrm{H}_{2} \mathrm{O}$ & EtOH & r.t. & $30-300$ & $75-94$ & [19] \\
\hline 12 & $\mathrm{Ce}\left(\mathrm{SO}_{4}\right)_{2} \cdot 4 \mathrm{H}_{2} \mathrm{O}$ & - & 120 & $15-25$ & $88-97$ & This work \\
\hline
\end{tabular}

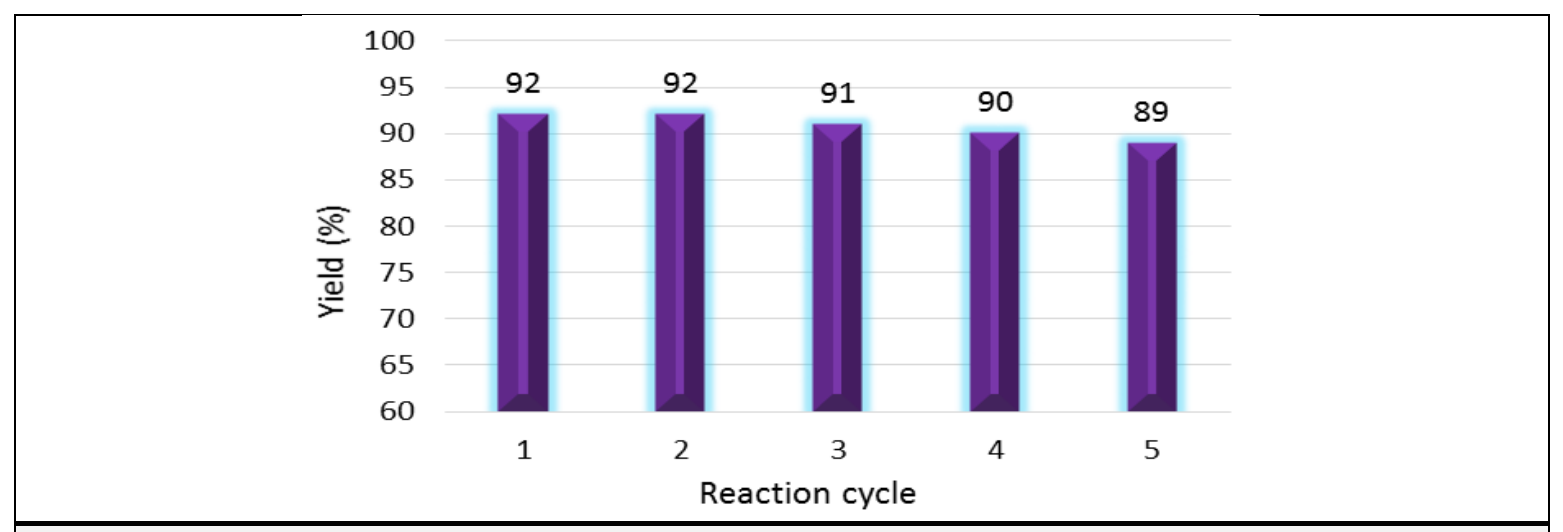

Figure 1. Effect of recycling on catalytic performance of $\mathrm{Ce}\left(\mathrm{SO}_{4}\right)_{2} \cdot 4 \mathrm{H}_{2} \mathrm{O}$ in the synthesis of $\mathbf{5 b}$ in the model reaction 


\section{Conclusion}

In conclusion, we have described a clean and efficient one-pot multicomponent condensation of dimedone, different aldehydes, ethyl acetoacetate and ammonium acetate for the synthesis of polyhydroquinolines utilizing Cerium (IV) sulfate tetrahydrate as a new heterogeneous catalyst. The catalyst could be recycled after a simple work-up, and used with only slight reduction in its catalytic activity. Furthermore, solventfree media, easy work-up, high yields, short reaction times and environmentally friendly conditions are other obvious advantages of this method.

\section{Acknowledgements}

The authors express their gratitude to the Islamic Azad University, Mashhad Branch for its financial support.

\section{References}

[1]. A. Nakhaei, A. Davoodnia, S. Yadegarian, Heterocycl. Lett., 2016, 6, 601-608.

[2]. C.S. Reddy, M. Raghu, Chin. Chem. Lett., 2008, 19, 775-779.

[3]. M. Rohaniyan, A. Davoodnia, A. Nakhaei, Appl. Organomet. Chem., 2016, 30, 626-629. [4]. G. Sabitha, G.K.K. Reddy, C.S. Reddy, J. Yadav, Tetrahedron Lett., 2003, 44, 41294131.

[5]. N.K. Ladani, D.C. Mungra, M.P. Patel, R.G.
Patel, Chin. Chem. Lett., 2011, 22, 14071410.

[6]. S.X. Wang, Z.Y. Li, J.C. Zhang, J.T. Li, Ultrason. Sonochem., 2008, 15, 677-680.

[7]. M.M. Heravi, H. Hamidi, N. Karimi, A. Amouchi, Adv. J. Chem. A, 2018, 1, 1-6.

[8]. S.B. Sapkal, K.F. Shelke, B.B. Shingate, M.S. Shingare, Tetrahedron Lett., 2009, 50, 1754-1756.

[9]. A. Khojastehnezhad, F. Moeinpour, A. Davoodnia, Chin. Chem. Lett., 2011, 22, 807810.

[10]. S. Sheik Mansoor, K. Aswin, K. Logaiya, S.P.N. Sudhan, Arab. J. Chem., 2017, 10, S546S553.

[11]. A. Kumar, R.A. Maurya, Tetrahedron Lett., 2007, 48, 4569-4571.

[12]. A. Heydari, S. Khaksar, M. Tajbakhsh, H.R. Bijanzadeh,J. Fluor. Chem., 2009, 130, 609-614. [13]. R. Surasani, D. Kalita, A.D. Rao, K. Yarbagi, K. Chandrasekhar, J. Fluor. Chem., 2012, 135, 91-96.

[14]. J.L. Donelson, R.A. Gibbs, S.K. De, J. Mol. Catal. A-Chem., 2006, 256, 309-311.

[15] S. Ko, C.-F. Yao, Tetrahedron, 2006, 62, 7293-7299.

[16]. M.M. Heravi, K. Bakhtiari, N.M. Javadi, F.F. Bamoharram, M. Saeedi, H.A. Oskooie, J. Mol. Catal. A-Chem., 2007, 264, 50-52.

[17]. L.M. Wang, J. Sheng, L. Zhang, J.W. Han, Z.Y. Fan, H. Tian, C.T. Qian, Tetrahedron, 2005, 61, 1539-1543. 
[18]. A. Zare, M. Dashtizadeh, M. Russ. J. Gen. Chem., 2017, 87, 1073-1078.

Merajoddin, Iran. Chem. Commun., 2015, 3, [32] A. Nakhaei, A. Davoodnia, A. Morsali, 208-217.

[19]. A. Somwanshi, Y.B. Pandit, A. Gholap, R. Res. Chem. Intermediat., 2015, 41, 7815Ghogare, S. Pandit, Iran. Chem. Commun., 2017, 5, 293-300.

[20]. S. Sajjadifar, Chem. Method., 2017, 1, 111.

[21]. S. Rezayati, R. Hajinasiri, Z. Hossaini, S. Abbaspour, Asian J. Green Chem., 2018, 2, 268-280.

[22]. M.M. Heravi, H. Abdi Oskooie, Z. Latifi, H. Hamidi, $A d v$. J. Chem. A, 2018, 1, 7-11.

[23]. I. Amini, K. Pal, S. Esmaeilpoor, A. Abdelkarim, Adv. J. Chem. A, 2018, 1, 12-31. [24]. H. Bakhshy, S.M.A. Rohani, P. Heydari, R. Asadi, Adv. J. Chem. A, 2018, 1, 32-38.

[25]. J. Ghodsi, A.A. Rafati, Y. Shoja, Adv. J. Chem. A, 2018, 1, 39-55.

[26] M.H. Nasirtabrizi, S.J. Mousavi, Adv. J. Chem. A, 2018, 1, 56-65.

[27] D. González-Calderón, C.A. GonzálezGonzález, A. Fuentes-Benítez, E. CuevasYáñez, D. Corona-Becerril, C. GonzálezRomero, Tetrahedron Lett., 2013, 54, 71647166.

[28] N.P. Selvam, P.T. Perumal, Tetrahedron lett., 2006, 47, 7481-7483.

[29] L. He, C.A. Horiuchi, Appl. Organomet. Chem., 1999, 13, 867-869.

[30] A. Nakhaei, Z. Nakhaei, Heterocycl. Lett., 2017, 7, 605-611.

[31] A. Nakhaei, A. Morsali, A. Davoodnia, 7826.

[33] A. Nakhaei, A. Davoodnia, S. Yadegarian, Iran. Chem. Commun., 2018, 6, 6-15.

[34] A. Nakhaei, S. Ramezani, S.J. ShamsNajafi, S. Farsinejad, Lett. Org. Chem., 2018, 15, 739-746.

[35] A. Nakhaei, Curr. Catal., 2018, 7, 72-78.

[36] A. Nakhaei, A. Davoodnia, A. Morsali, Russ. J. Phys. Chem. A, 2018, 92, 271-279.

[37] A. Nakhaei, S. Farsinejad, S. Ramezani, Curr. Green Chem., 2017, 4, 130-136.

[38] A. Nakhaei, Russ. J. Gen. Chem., 2017, 87, 1850-1856.

[39] A. Nakhaei, H. Nakhaei, Heterocyclic Letters, 2018, 8, 27-33.

[40] A. Nakhaei, Heterocycl. Lett., 2017, 7, 967-973.

[41] A. Nakhaei, S. Ramezani, Heterocycl. Lett., 2017, 7, 1035-1043.

[42] A. Nakhaei, Z. Nakhaei, Heterocycl. Lett., 2017, 7, 565-572.

[43] A. Nakhaei, S. Shojaee, E. Yaghoobi, S. Ramezani, Heterocycl. Lett., 2017, 7, 323331.

[44] A. Nakhaei, Z. Nakhaei, Iran. J. Org. Chem., 2017, 9, 2135-2144.

[45] A. Nakhaei, S. Yadegarian, Iran. J. Org. Chem., 2017, 9, 2057-2065.

[46] Y. Mirzaie, J. Lari, H. Vahedi, M. Hakimi, A. Nakhaei, A. Rezaeifard, J. Mex. Chem. Soc., 
2017, 61, 35-40. 1497-1503.

[47] N.G. Khaligh, Chinese J. Catal., 2014, 35,

How to cite this manuscript: Elham kazemi, Abolghasem Davoodnia*, Samira Basafa, Ahmad Nakhaei*, Niloofar Tavakoli-Hoseini, Investigating Effect of Cerium (IV) Sulfate Tetrahydrate as Reusable and Heterogeneous Catalyst for the One-pot Multicomponent Synthesis of Polyhydroquinolines, Adv. J. Chem. A, 2018, 1(2), 96-104. 